\title{
NOVEL CONCEPT OF SATELLITE MANOEUVRE PLANNING USING GRAPH THEORETICAL TECHNIQUES
}

\author{
Ciara N. McGrath, ${ }^{\star}$ Ruaridh A. Clark, ${ }^{\dagger}$ and Malcolm Macdonald ${ }^{\ddagger}$
}

\begin{abstract}
Manoeuvrable, responsive satellite constellations that respond to real time events could provide data on demand for time-critical tasks, such as disaster monitoring and relief efforts. The authors demonstrate the feasibility of such a system by expanding on a fully analytical method for designing responsive spacecraft manoeuvres using low thrust propulsion. These manoeuvres are perceived as a graph that enables efficient exploration and optimised selection of favourable routes that achieve mission goals while highlighting resilience and redundancy in the mission's execution. A case study is presented that considers four satellites required to provide flyovers of two targets, with an associated graph of 10839 possible manoeuvres. Investigation of the graph highlights that a good, but not minimum time, solution can allow the system to perform well, while also providing greater resilience to changes in mission priorities and errors in execution. This analytical approach enables operators to trade-off between a loss of time by using only one satellite versus the disruption of moving multiple satellites for a potentially faster response. The impact of varying mission capabilities, such as using fewer satellites, smaller swath width or less propellant, can be evaluated by reducing the graph without recalculating manoeuvre options.
\end{abstract}

Keywords: Constellation; Manoeuvre; Graph; Responsive

\section{INTRODUCTION}

Interest in responsive satellite systems is growing as terrestrial applications increasingly necessitate the use of real time, on demand data (Voigt, et al., 2016; Santilli, et al., 2018; Gopinath, 2015). Current state of the art satellite systems, such as those operated by Planet, Inc. (Boshuizen, et al., 2014) and Spire Global, Inc. (Platzer, et al., 2015; Buchen, 2015), cannot manoeuvre and, as such, would require thousands of satellites provide global, realtime coverage. This would be both impractical and financially prohibitive with potentially severe implications for our already congested space environment (Morin, 2019; Skinner, et al., 2019). Successful implementation of manoeuvrable satellite systems will address this issue by reducing the number of spacecraft needed to provide on demand information for time critical applications, such as disaster response. However, to ensure efficient operation of such a system, an understanding of the capabilities and limitations of manoeuvrable spacecraft is required, as is a method for analysing and comparing the multitude of distinct manoeuvre options possible to ensure an efficient concept of operations for the system.

Previous research by the authors has developed a fast and accurate method of planning spacecraft manoeuvres using low thrust propulsion that can facilitate rapid analysis of responsive scenarios involving numerous satellites, targets, and ground stations (McGrath \&

\footnotetext{
${ }^{*}$ Research Associate, Department of Mechanical and Aerospace Engineering, University of Strathclyde, 16 Richmond St, Glasgow G1 1XQ, UK, ciara.mcgrath@strath.ac.uk.

$\dagger$ Research Associate, Department of Mechanical and Aerospace Engineering, University of Strathclyde, 16 Richmond St, Glasgow G1 1XQ, UK, ruaridh.clark@strath.ac.uk.

\$ Professor, Department of Mechanical and Aerospace Engineering, University of Strathclyde, 16 Richmond St, Glasgow G1 1XQ, UK, malcolm.macdonald.102@strath.ac.uk.
} 
Macdonald, 2019; McGrath, 2018). This method uses general perturbation techniques and thus can produce a large number of solutions extremely quickly whilst maintaining a high degree of accuracy. The method can provide the user with a full overview of all the eligible manoeuvre options for flying over a region of interest on the Earth. These options will vary in terms of the change in velocity $(\Delta \mathrm{V})$ required for the manoeuvre, as well as the time taken for the manoeuvre and the look angle to the target at flyover. As such, the ability to consider all options is extremely valuable, allowing the operator to trade off each solution and identify those that best align with their unique mission priorities. This previous work by McGrath \& Macdonald, 2019 demonstrated that reconfiguring a constellation of 24 satellites could provide increased persistence of coverage of 1.6 - 10 times compared to a traditional, non manoeuvring constellation, depending on the latitude of the target region. For the scenario considered in this previous work, it was predicted that up to 12 targeting reconfigurations could be performed. However, these prior analyses selected the reconfiguration manoeuvres by considering each reconfiguration independently; in fact, manoeuvres selected early in the mission will affect the choices available in the future and thus, for truly efficient operations, an assessment is required that considers the full sequence of manoeuvres necessary to achieve the mission goals.

This article addresses the challenge of manoeuvre planning for responsive spacecraft constellations by using the previously developed method of low-thrust spacecraft manoeuvre propagation to create an expansive trade space of manoeuvre options. This trade space is represented as a graph that can be explored to obtain insights into the capabilities of the responsive system and to devise a concept of operations that considers the entire operational scenario. The use of the previously derived fast method of manoeuvre calculation allows for large graphs, encompassing thousands of manoeuvres, to be generated.

A graph capturing all possible manoeuvre options, where each option is represented as an edge, can comprise of many of thousands of nodes that each represent a flyover of a target. When edges are supplied with a weighting that captures some property of a manoeuvre, such as time taken or $\Delta \mathrm{V}$ required, then an optimal path through the graph can emerge. Manually identifying effective routes in large graphs is not always feasible, but shortest path algorithms, such as Dijkstra's algorithm (Dijkstra, 1959), or tree-search methods (Silver, et al., 2016), including branch and bound algorithms (Morrison, et al., 2016) can efficiently identify these paths to inform manoeuvre decisions. Dijkstra's shortest path algorithm has previously been applied to space applications to design efficient routing processes for data transfer through spacecraft constellations with intersatellite communication capabilities (Gnawali, et al., 2005; Lowe \& Macdonald, 2016). Branch and bound solution algorithms have also been applied to space applications, such as to define an optimal targeting schedule for an agile Earth observing satellite (Chu, et al., 2017). These applications focus on minimising cost and/or maximising profit by identifying the most favourable 'path' through the system. However, these metrics, while useful, are limited, especially when considering a responsive satellite system, where a shortest path might become unusable if there is a change in mission priorities, such as a need to conserve $\Delta \mathrm{V}$ or reduce flyover time. Therefore, a route through the graph that presents many good options rather than a single optimal option may be preferable. The proposed method enables a trade off between finding a short route through the graph and ensuring that there are many good options if the route needs to be altered.

This combination of graph-theoretical techniques with rapid orbit propagation and manoeuvrability is a novel approach to responsive satellite operations that offers a new way of exploring and analysing space missions. The speed of the technique makes it ideal for mission design and trade space exploration, by providing an efficient means of assessing the expected performance of a responsive spacecraft constellation architecture. It also provides an efficient methodology for use in responsive operational planning and enables the cost of responsiveness, in terms of propellant requirements, to be assessed and quantified, so that an 
informed decision can be made considering this cost against the benefits of increased, or more timely, coverage. Furthermore, by providing a full overview of all manoeuvre options, rather than focusing on determining the shortest path solution, the proposed method enables an operator to assess the resilience and redundancy of the possible options.

This article is constructed as follows. The Method Section (Section 2) shall introduce how the manoeuvre sequence graph is constructed - highlighting the different types of manoeuvre - and how this graph is searched and analysed. The Case Study Section (Section 3) shall then introduce the scenario of overflying two national parks to perform fire monitoring observations. The Results Sections (Sections 4 and 5) explore the added insights, provided by the graph analysis, that would be available to an operator in this mission scenario for differing mission resources and priorities.

\section{METHOD}

\subsection{Problem Statement}

To understand the motivation behind this work, first consider a single, manoeuvrable satellite that is required to sequentially fly over two targets, A and B. There are a multitude of manoeuvres that can be employed to flyover the targets, each with a different $\Delta \mathrm{V}$ cost, manoeuvre time, and resultant look angle to the target (McGrath \& Macdonald, 2019). The choice of manoeuvre will determine the spacecraft's new orbit parameters, which will in turn determine the subsequent manoeuvre options. This is presented in Figure 1, where each possible flyover of each target is considered as distinct, due to the difference in orbit parameters at the time of flyover.

In Figure 1, there are three possible manoeuvre options for flying over target $\mathrm{A}$ and the required $\Delta \mathrm{V}$ is indicated for each manoeuvre. For the purposes of this example, minimising $\Delta \mathrm{V}$ is assumed to be the only operational goal; manoeuvre time and look angle at flyover are also considered but only in terms of providing operational constraints (e.g. maximum values allowable), such that the manoeuvres shown are those that meet the selected criteria. Consider the first manoeuvre to flyover target $\mathrm{A}$; in considering this stage of manoeuvring alone, it is clear from Figure 1 that the uppermost manoeuvre, requiring $1 \mathrm{~m} / \mathrm{s} \Delta \mathrm{V}$, is the minimum $\Delta \mathrm{V}$ manoeuvre. However, from this point there are no suitable manoeuvres available to provide a subsequent flyover of target $\mathrm{B}$. The central manoeuvre to flyover target A requires $3 \mathrm{~m} / \mathrm{s} \Delta \mathrm{V}$ and is the next best manoeuvre for the first stage, however the next manoeuvre to flyover target $\mathrm{B}$ has a very high $\Delta \mathrm{V}$ cost. Indeed, in this scenario, choosing the highest $\Delta \mathrm{V}$ manoeuvre for the first stage to flyover target $\mathrm{A}$ will minimise the $\Delta \mathrm{V}$ required for the full scenario. The minimum $\Delta \mathrm{V}$ path for this scenario is shown as a dashed green arrow in Figure 1. This illustrates the need to consider the full operational scenario, rather than selecting manoeuvres for each stage of the mission independently.

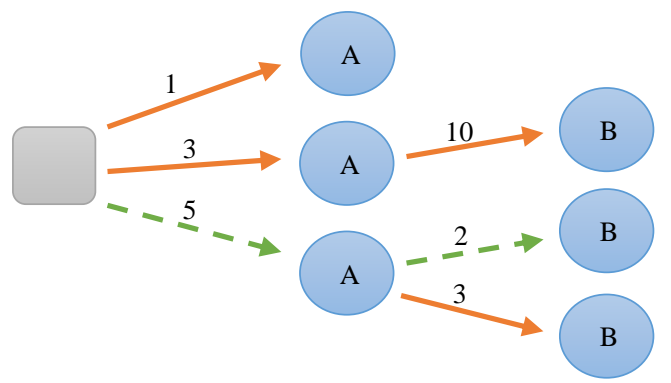

Figure 1: Scenario for sequential flyover of targets $A$ then $B$ from an initial system state, with each possible manoeuvre option represented by a single arrow. Numbers represent the $\Delta \mathrm{V}$ required for each manoeuvre. The minimum $\Delta \mathrm{V}$ path is shown as a dashed green arrow. 
A more complex scenario can be envisaged, in which two satellites are available to manoeuvre and fly over two targets, A and B, but the flyovers can occur in any order. Satellite 1 or 2 could flyover both targets, in either order, or each satellite could flyover one target. Additionally, for each manoeuvre there are a variety of possible options, which differ in $\Delta \mathrm{V}$, manoeuvre time and look angle to target at flyover; this is visualised in Figure 2. It is clear that this presents a greater challenge than the previous example, though it is much simpler than the operational scenarios expected of a real responsive constellation. Inspection of Figure 2 identifies the minimum $\Delta \mathrm{V}$ solution as the case in which Satellite 1 overflies Target A followed by Target B, at a cost of $2 \mathrm{~m} / \mathrm{s}$. However, once Satellite A has performed the first manoeuvre to flyover Target A, there is only one manoeuvre option to flyover Target $\mathrm{B}$; if this manoeuvre is no longer suitable (for example if a faster flyover becomes necessary), or no longer viable (for example if a slight error in manoeuvre execution results in the subsequent manoeuvre becoming too costly) then there are no alternative manoeuvre options available to complete the mission. An alternative option is for Satellite 2 to flyover Target B and then Target $\mathrm{A}$; this requires a minimum $\Delta \mathrm{V}$ of $4 \mathrm{~m} / \mathrm{s}$ but is a more resilient option as there is an additional manoeuvre option to flyover Target A should the first option become unusable, for any reason. A third option is for Satellite 1 to flyover Target B and Satellite 2 to flyover Target A, with both spacecraft manoeuvring simultaneously, requiring a total $\Delta \mathrm{V}$ of 3 $\mathrm{m} / \mathrm{s}$. This would result in greater disruption to the constellation by requiring two spacecraft to manoeuvre, but provides redundancy as subsequent manoeuvres are available for both spacecraft to flyover the targets should there be an issue with the execution of the first manoeuvres. This illustrates the benefit of considering all possible options, rather than simply identifying the shortest path solution.

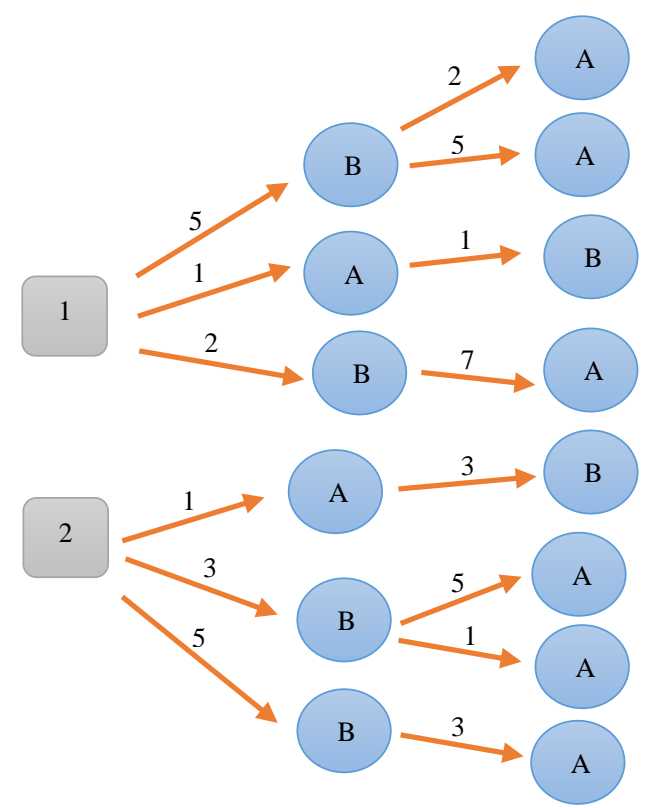

Figure 2: Scenario where two satellites can flyover targets A and B in any order, with each possible manoeuvre option represented by a single arrow. Numbers represent the $\Delta \mathrm{V}$ required for each manoeuvre.

\subsection{Representing the Scenario as a Graph}

In order to consider all the possible options to complete the task of flying over multiple targets, in any order, with multiple spacecraft available for tasking, it is helpful to visualise the scenario as a graph. This is similar to the decision trees shown in Figure 2, where the 
possible flyovers of each target can be considered as nodes and the manoeuvres can be considered as edges. However, the graph representation shall now incorporate multiple satellites to enable concurrent analysis of all satellite manoeuvres for target flyover.

The flyovers of targets are defined as nodes in the graph, with the location of these targets defined in terms of their latitude and longitude. The initial positions of all satellites in the constellation are defined in terms of their Keplerian orbital elements (Bate, et al., 1971) (i.e. semi major axis, $a$; eccentricity, $e$; inclination, $i$; right ascension of the ascending node (RAAN), $\Omega$; argument of perigee, $\omega$; and mean anomaly, $M$ ). Additionally, the Julian date of the epoch must be defined to orient the constellation relative to the Earth. An edge is created if there exists a manoeuvre that takes a satellite from its initial position and results in it overflying the location of a target. In order to establish a realistic search space, constraints are placed on the maximum manoeuvre time, $\Delta \mathrm{V}$ for a single manoeuvre, and on the maximum look angle to target, which corresponds to the look angle of the spacecraft instrument. Apart from the number of satellites and targets being considered, these constraints will have the largest impact on the time required to generate the graph.

The first node in the graph, node 0 , is the root node. From the initial conditions at epoch, all possible manoeuvres for each satellite to fly over each target beginning from their initial locations are calculated. For each manoeuvre, the position of the satellite that has manoeuvred at the time the manoeuvre is completed is recorded. This flyover forms a new node in the graph, linked to node 0 by a directed edge representing the manoeuvre. For each manoeuvre, the position of the satellite that has manoeuvred at the time the manoeuvre is completed is recorded. This flyover forms a new node in the graph, linked to node 0 by a directed edge representing the manoeuvre. This edge will hold the manoeuvre time and $\Delta \mathrm{V}$ parameters as weightings. The nodes will hold the information of which target has been seen, which satellite has manoeuvred, the time at which the manoeuvre is completed, the look angle to the target at flyover, and the position of the manoeuvring spacecraft at this time. This first set of possible manoeuvres will be referred to as Stage 1 manoeuvres.

The set of nodes, connected to node 0 by Stage 1 manoeuvres, are used as the starting conditions for a new set of manoeuvres. For the satellite that has just manoeuvred, the conditions at the end of the previous manoeuvre are used as the starting conditions for the manoeuvre calculations. For all other spacecraft, the starting conditions for the manoeuvre calculations are determined by propagating the spacecraft for the total time elapsed through the preceding edges. These new manoeuvres, defined as Stage 2, exist if a manoeuvre exists that will flyover a target that has not yet been seen on the path that leads to the current node. This process is continued with new nodes and manoeuvres until the number of stages (manoeuvres) is the same as the number of targets.

The scalability of the presented method is highly dependent on the size of the graph being generated. For the case outlined, in which a single flyover of each target is required, the graph will be a tree graph with the depth of the tree $(d)$ dictated by the number of targets. The branching factor $(b)$ will be dictated by number of satellites $(s)$, the number of targets $(t)$ and the number of possible manoeuvre options at each node such that $\max (b)=s t\left(\frac{\Delta V_{\max }}{\Delta V_{\text {step }}}\right)$, where $\Delta V_{\text {max }}$ is the maximum $\Delta \mathrm{V}$ considered for a single manoeuvre and $\Delta V_{\text {step }}$ is the increments of $\Delta \mathrm{V}$ at which each possible manoeuvre is calculated. If a suitable manoeuvre does not exist for a certain combination of satellite/target/ $\Delta \mathrm{V}$ then it is not added to the graph and $b<\max (b)$ for the node in question. The maximum time complexity for enumerating the full tree is thus $O\left(M b^{d}\right)$ (Morrison, et al., 2016) where $M$, in this case, is the time complexity of the calculation to generate a single manoeuvre option. Due to the power law present in this enumeration time, the number of targets will have a large impact on graph generation as that determines the depth of the tree $(d)$, while the number of satellites considered will have a relatively small impact. This implies the suitability of the presented 
method for large constellations of spacecraft requiring flyovers of a limited number of targets. In this paper we consider two targets, while adopting a large swath width and a wide range of manoeuvre time and $\Delta \mathrm{V}$. When considering more targets, it may be necessary to constrain the swath width and manoeuvre parameters to ensure computational feasibility.

2.2.1 Manoeuvre Calculations. Generating the graph is the most resource intensive aspect of the presented method and is most likely to limit the scalability of the method; by comparison, the time required for graph analysis is negligible. Therefore, it is important to choose a method of manoeuvre calculation that can be executed efficiently. Herein, the fast general perturbation method previously derived by the authors (McGrath \& Macdonald, 2019; McGrath, 2018) is used to calculate the manoeuvre options for all scenarios. This method assumes the use of low thrust propulsion for circular to circular, coplanar manoeuvres and considers gravitational perturbations due to an oblate central body up to the order of $J_{2}$. Atmospheric drag is assumed to be compensated for throughout and, as such, the spacecraft maintains a constant altitude when not actively manoeuvring; any $\Delta \mathrm{V}$ required to maintain this altitude is included in the $\Delta \mathrm{V}$ cost associated with a manoeuvre, for both the manoeuvring and nonmanoeuvring spacecraft. The manoeuvres only directly change the altitude of the satellite, but this in turn causes a change in the RAAN and argument of latitude (AoL) due to the variation in orbit period and central body perturbations. For all cases herein, the propagation of any nonmanoeuvring satellites is done using the same method, but with no manoeuvres performed.

\subsection{Manoeuvre Types}

Considering the full manoeuvre sequence, there are three different types of possible scenarios. Same satellite manoeuvres are those cases in which the same satellite performs all the manoeuvres on a single path sequentially. This may have operational benefits as only one satellite must be manoeuvred for the mission to be completed. Separate sequential manoeuvres are those cases in which different satellites manoeuvre at different stages of the manoeuvre sequence. This may produce a lower $\Delta \mathrm{V}$ solution, but may increase operational complexity and/or increase the total manoeuvre time. These manoeuvres may also provide redundancy in the sequence, as they enable the operator to delay manoeuvre execution until later in the sequence. This could be of value if, for example, the need to flyover a target is not yet confirmed. Separate simultaneous manoeuvres are those in which two or more separate satellites manoeuvre simultaneously to provide flyovers of different targets. An alternative method is required to include separate simultaneous manoeuvres in the graph and is described in Section 2.3.1.

2.3.1 Separate Simultaneous Manoeuvres The manoeuvre-target graph described thus far can only represent operational scenarios in which manoeuvres are performed sequentially; this may include same satellite manoeuvres, or manoeuvres by different spacecraft, but it assumes that there is no overlap in time among these manoeuvres. In reality, it may be desirable to move two or more satellites simultaneously to flyover multiple targets. To account for this, manoeuvres from one stage, in the form of edges, can be copied from the graph and 'transplanted' onto nodes in the subsequent stage. This is referred to throughout as separate simultaneous manoeuvres and illustrated in Figure 3, where Stage 2 manoeuvres of the same colour and style as those in Stage 1 are copies of Stage 1 manoeuvres.

When transplanting manoeuvres, the $\Delta \mathrm{V}$ assigned to the edge will be the same as that for the original manoeuvre, but the time will vary, as the time along both edges must sum to give the time of the longest manoeuvre on the path; this is necessary to account for the fact that both manoeuvres happen in tandem. As such, if the time of the first manoeuvre, $t_{0}$, is less than the time required for the transplanted manoeuvre, $t_{\text {trans }}$, then the time assigned to the new edge, $t_{\text {new }}$, will be the difference between the two times. If $t_{0}$ is greater than $t_{\text {trans }}$, then the new assigned time should be zero; however, for implementation it is necessary to assign a 
small time weighting to the edge, as edges with a weighting of zero will be assumed to not exist. Mathematically this can be expressed as

$$
\Delta V_{\text {new }}=\Delta V_{\text {trans }}, \text { and } t_{\text {new }}=\left\{\begin{array}{l}
t_{\text {trans }}-t_{0}, t_{\text {trans }}>t_{0} \\
t_{\text {new }} \ll t_{0}, t_{\text {trans }} \leq t_{0}
\end{array} .\right.
$$

It must additionally be ensured that the transplanted manoeuvres are performed using a different satellite than the manoeuvre they are being transplanted to. For the illustrative example shown in Figure 3, three different spacecraft, indicated by different coloured and styled arrows, can initially perform manoeuvres to flyover targets A or B. To account for the option of these manoeuvres occuring simultaneously, two manoeuvres are transplanted onto the first path. Here, $t_{4}=\left\{\begin{array}{c}t_{2}-t_{1}, t_{2}>t_{1} \\ t_{4} \ll t_{1}, t_{2} \leq t_{1}\end{array}\right.$, and $t_{5}=\left\{\begin{array}{c}t_{3}-t_{1}, t_{3}>t_{1} \\ t_{5} \ll t_{1}, t_{3} \leq t_{1}\end{array}\right.$.

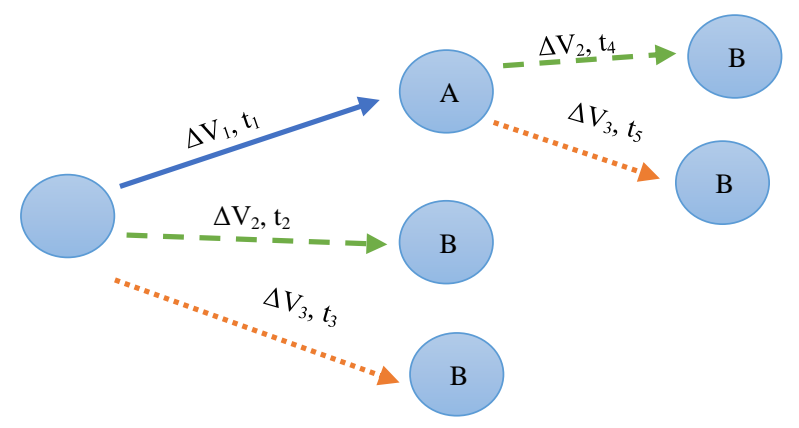

Figure 3: Transplanting of edges to represent separate manoeuvres. Letters indicate targets. Edges with the same colour and style are transplanted copies.

\subsection{Analysing the Graph}

Prior to analysis, the graph can be reduced based on a number of operational constraints by removing any nodes and edges that fall outside these criteria. For example, if there is a minimum required look angle to target, then any nodes that do not meet this criterion and the paths that extend from these nodes can be removed from the search space. Similarly, if there is a maximum time that the mission must be completed in, then any paths that exceed this time can be removed. In this way, once the full graph has been generated, various sub-cases can be analysed to assess the impact of varying mission parameters, without the need to generate a new graph.

Once the graph has been reduced, if required, analysis of the scenario can be performed. For the cases examined herein, this is done by applying Dijkstra's algorithm (Dijkstra, 1959) to find the shortest path through the graph; however, alternative graph search methods could be used. The choice of weighting parameters applied to the edges of the graph (e.g. $\Delta \mathrm{V}$, time, or a utility function capturing multiple parameters) will determine the outcome of Dijkstra's algorithm. For example, assigning the manoeuvre $\Delta \mathrm{V}$ as a weight to each edge will mean that Dijkstra identifies the combination of manoeuvres that will require the minimum total $\Delta \mathrm{V}$ across all spacecraft manoeuvres. Similarly, using manoeuvre time to weight each edge provides the combination of manoeuvres that complete the mission in the shortest total time.

Dijkstra's algorithm can provide a shortest path solution when the graph is weighted and the path must include flyovers of all targets. However, the real benefit of representing the system as a graph in this article is the ability to identify how many near optimal options and 
routes through the graph exist. If the analysis perfectly represented the satellite system, and no change in mission priorities were to occur then the shortest path analysis would be sufficient. Given that these criteria cannot be taken for granted, there is value in identifying a manoeuvre that not only provides a short path through the graph but also leads to a range of other short path options. A path that leads to a larger range of manoeuvre options is more likely to be able to accommodate a change in circumstance, such as shifting mission priorities or manoeuvre execution error. This resilience in the manoeuvre path is in contrast to a path with only one manoeuvre option, after the first manoeuvre, where any change could lead to the second manoeuvre becoming infeasible.

The other benefit of a graphical analysis of the system is the ability to select a route where redundancy exists. For example, a short path separate simultaneous manoeuvre can be performed where it is known that a short path same satellite manoeuvre also exists. Therefore, in the case where providing a minimum time flyover is the objective, both the separate satellite and same satellite manoeuvre can be performed to add redundancy in flying over the second target; should the separate simultaneous manoeuvre fail to provide the required coverage for any reason, the same satellite manoeuvre can subsequently be used to provide an additional opportunity for data collection.

\section{CASE STUDY}

To demonstrate the proposed method of responsive constellation manoeuvre planning, a case study is investigated in which a constellation of four satellites is required to flyover two targets in any order. The constants and mission parameters used for this analysis are given in Table 1 and Table 2, respectively. It is assumed that the spacecraft have a conical field of view and hence the swath width represents the diameter of a circular region on the ground. If a target is located within this circular field of view it is considered to be visible. The targets are selected as the centre of the Cairngorms National Park and Yosemite National Park as shown in Figure 4. These areas were selected due to their propensity for fire outbreaks where responsive satellites could be tasked to monitor wildfire outbreaks. Cairngorms National Park is a region of spectacular beauty in Scotland that is of high conservation importance due to its unique flora and fauna; however, it is at increasing risk of fire outbreak (Carver, et al., 2008; Gray \& Levy, 2009). Yosemite National Park is the third most visited national park in the world with almost 4 million visitors annually, but it has a very high fire risk in the drier months (National Park Service, 2017; Lutz, et al., 2009; Kane, et al., 2014). The manoeuvre calculation method described in Section 2 is used, with manoeuvres calculated at increments of $1 \mathrm{~m} / \mathrm{s} \Delta \mathrm{V}$ up to the maximum allowable.

Table 1. Constants.

\begin{tabular}{c|c|c}
\hline Parameter & Value & Unit \\
\hline Gravitational Parameter & $3.986 \times 10^{14}$ & $\mathrm{~m}^{3} / \mathrm{s}^{2}$ \\
\hline Mean radius of Earth & 6371 & $\mathrm{~km}$ \\
\hline Coefficient of J2 for Earth & 0.0010827 & - \\
\hline Angular velocity of Earth & $7.29212 \times 10^{-5}$ & $\mathrm{rad} / \mathrm{s}$ \\
\hline Earth flattening & $3.35281 \times 10^{-3}$ & - \\
\hline Propagation time step & 10 & $\mathrm{sec}$ \\
\hline
\end{tabular}


Table 2. Mission Parameters.

\begin{tabular}{c|c|c}
\hline Parameter & Value & Unit \\
\hline Time and date at epoch & $00: 001 / 1 / 1991$ & - \\
\hline Julian date at epoch & 2448257.5 & - \\
\hline Target 1 latitude & 57.120 & $\mathrm{deg}$ \\
\hline Target 1 longitude & -3.645 & $\mathrm{deg}$ \\
\hline Target 2 latitude & 37.835 & $\mathrm{deg}$ \\
\hline Target 2 longitude & -119.545 & $\mathrm{deg}$ \\
\hline Max. time per manoeuvre & 7 & $\mathrm{days}$ \\
\hline Max. $\Delta$ V per manoeuvre & 10 & $\mathrm{~m} / \mathrm{s}$ \\
\hline Instrument swath & 100 & $\mathrm{~km}$ \\
\hline
\end{tabular}

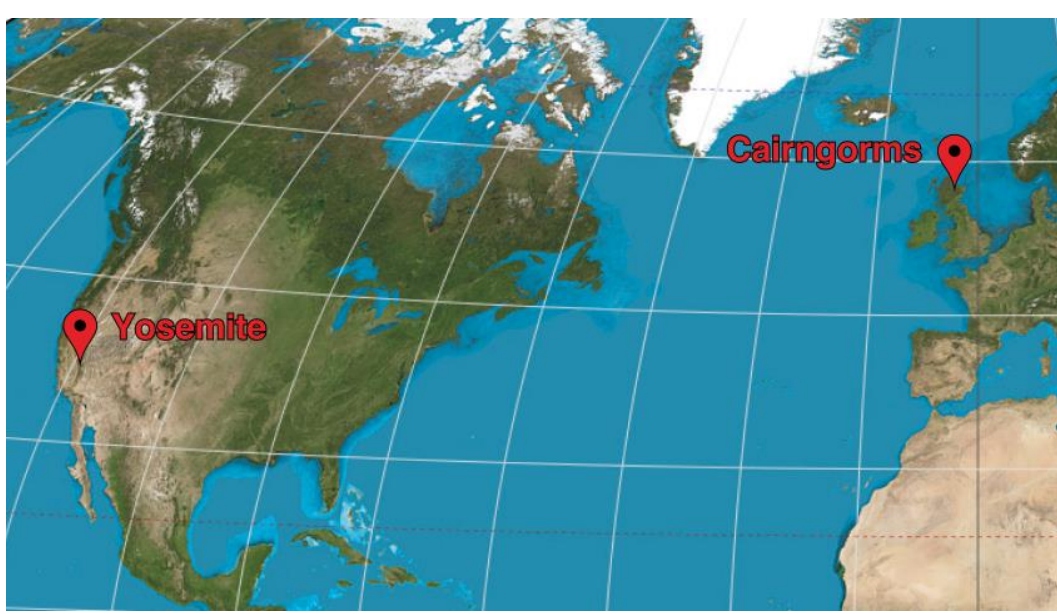

Figure 4: Map highlighting both flyover targets.

\subsection{Spacecraft Description}

For this case study, the constellation is assumed to be made up of four CubeSats equipped with the electrospray propulsion system developed by the Massachusetts Institute of Technology (Krejci, et al., 2017; Mier-Hicks \& Lozano, 2017). These propulsion systems are highly efficient and capable of producing sufficient acceleration to enable constellation reconfiguration (McGrath \& Macdonald, 2019). CubeSats are constrained in volume, mass and power; however, the recent miniaturisation of components has enabled even small spacecraft to deliver valuable Earth observation data. A prime example are the Doves launched by Planet, which can provide images of the Earth with resolutions as low as 3 metres (Boshuizen, et al., 2014). The orbit parameters for the spacecraft to be considered are selected arbitrarily, with the constraint that the orbit inclination selected would allow both targets to be viewed by all spacecraft. The orbit and spacecraft parameters used in this analysis are given in Table 3 . 
Table 3. Spacecraft parameters.

\begin{tabular}{c|c|c}
\hline Parameter & Value & Unit \\
\hline Mass & 3 & $\mathrm{~kg}$ \\
\hline Cross sectional area & 0.03 & $\mathrm{~m}$ \\
\hline Coefficient of drag & 2.2 & - \\
\hline Thrust & $3.5 \times 10^{-4}$ & $\mathrm{~N}$ \\
\hline Orbit altitude & 542.857 & $\mathrm{~km}$ \\
\hline $\begin{array}{c}\text { Orbit inclination } \\
\begin{array}{c}\text { Walker formation (Walker, } \\
\text { 1977) }\end{array}\end{array}$ & 60 & $\mathrm{deg}$ \\
\hline
\end{tabular}

Analysing all the possible manoeuvres to complete the proposed mission produces a graph of 10840 nodes, where each node represents the system state immediately after a target flyover. One node (Node 0) represents the initial system state at epoch. This Node 0 is connected to 118 nodes that represent the system state after flyover of one target (i.e. Stage 1). These 118 nodes, in turn, are connected to 10721 nodes (i.e. Stage 2). Of these, 6157 nodes represent the system state after a set of sequential manoeuvres, while the other 4564 nodes are at the end of transplanted edges, representing a set of separate simultaneous manoeuvres. Generating the full graph of results takes approximately 1.7 minutes on a laptop computer running Windows 10 with 16 GB of RAM.

\section{RESULTS FOR FULL GRAPH}

In considering the possible manoeuvre options, it is important to consider the performance of the full manoeuvre sequence, in terms of time and/or $\Delta \mathrm{V}$ usage. Additionally, it is useful to assess if redundancy or resilience can be added to a manoeuvre sequence.

A manoeuvre sequence can be identified as having redundancy if it has separate sequential and/or separate simultaneous manoeuvres available, such that other satellites can fulfil the mission if tasked at either Stage 1 or Stage 2. It should be noted that a satellite cannot perform both a separate simultaneous manoeuvre and then a separate sequential manoeuvre, therefore when assessing redundancy only the manoeuvre type with the largest number of options is considered.

A manoeuvre sequence is more resilient if each satellite capable of performing a Stage 2 manoeuvre has connections to the next target for a range of $\Delta \mathrm{V}$ inputs; hence, more connections from a node represents a wider range of $\Delta \mathrm{V}$ that can be used to reach the target and the more resilient this manoeuvre sequence is to execution uncertainty or changes in mission priorities.

\subsection{Minimum Time Solution}

For the case study in Section 3, the Dijkstra's algorithm identified the shortest path that completes both target flyovers as taking 2 days 14 hours and 11 minutes (2.5913 days) and requiring $7.037 \mathrm{~m} / \mathrm{s} \Delta \mathrm{V}$. This shortest path involves the use of a separate sequential manoeuvre, i.e. manoeuvres occur sequentially but with different spacecraft employed in Stage 1 and Stage 2. 
A shortest path algorithm can only provide limited information about the overall scenario and the manoeuvre possibilities within it. However, restricting the graph to consider only manoeuvres with times close to that of the shortest path solution can provide greater insights; in this case the viable manoeuvre combinations are taken to be those with less than $10 \mathrm{~m} / \mathrm{s} \Delta \mathrm{V}$ in total that complete both target flyovers within 10 minutes of the time taken by the shortest path. In Figure 5a, the number of same satellite manoeuvre options are detailed for the shortest time manoeuvre sequences, which reveals that a same satellite manoeuvre can accomplish the mission with less than a 1 second difference in mission time (2.5913 days). A same satellite manoeuvre sequence can be an attractive option for an operator as there is less disruption to the constellation with only one satellite being manoeuvred.

Figure 5a also displays that the fastest same satellite solution has 8 same satellite manoeuvre options in Stage 2, which use varying amounts of $\Delta \mathrm{V}$ but all complete the mission within 3.5 minutes of each other. In this way the graph can highlight resilient manoeuvres, as the other fast, same satellite manoeuvre options have fewer Stage 2 manoeuvres available; a consequence of manoeuvres not being possible within the time limit and the range of feasible $\Delta \mathrm{V}$.

It should be noted that graphs, such as those displayed in Fig. 5, enable an operator to highlight Pareto dominance, where a Pareto dominant solution exists if it improves performance for at least one priority metric while not reducing performance according to any other priorities. For two priority metrics, defined as fast target coverage and number of viable $\Delta \mathrm{V}$ manoeuvres after the first manoeuvre, then the most options solution in Fig. 5a would be Pareto dominant. The difference in time between the most options solution and the shortest path solution is negligible in a satellite observation application, but there is a clear dominance in the number of same satellite manoeuvres.

Separate simultaneous and separate sequential manoeuvres both use two satellites to overfly both targets. This is a more disruptive approach but can ensure targets are covered with redundancy available. In Figure 5b, the shortest path manoeuvre sequence has both separate simultaneous and separate sequential manoeuvres available; therefore, if desired, a backup satellite could be tasked at both stages of the manoeuvre sequence while still guaranteeing the fastest response time. It is worth noting that the same satellite cannot be involved in both a separate simultaneous and separate sequential manoeuvre in a real scenario. Therefore when assessing the no. of separate satellite manoeuvre options in Figure $5 \mathrm{~b}$ for both separate manoeuvre types the largest number of options was selected from one of the two types rather than summing all of the options. As in the case of same satellite manoeuvres, a greater number of manoeuvre options corresponds to greater resilience by providing a range of possible manoeuvre options.

The question of Pareto dominance occurs again with Fig. 5b, but to determine dominance of the most options solution over the shortest path there are two aspects that would need to be determined. Firstly, whether a flyover occurring 34 seconds later constitutes a reduction in performance and, secondly, whether having many separate simultaneous manoeuvres is an advantage as all satellites would have to be tasked before the first manoeuvre. 
a

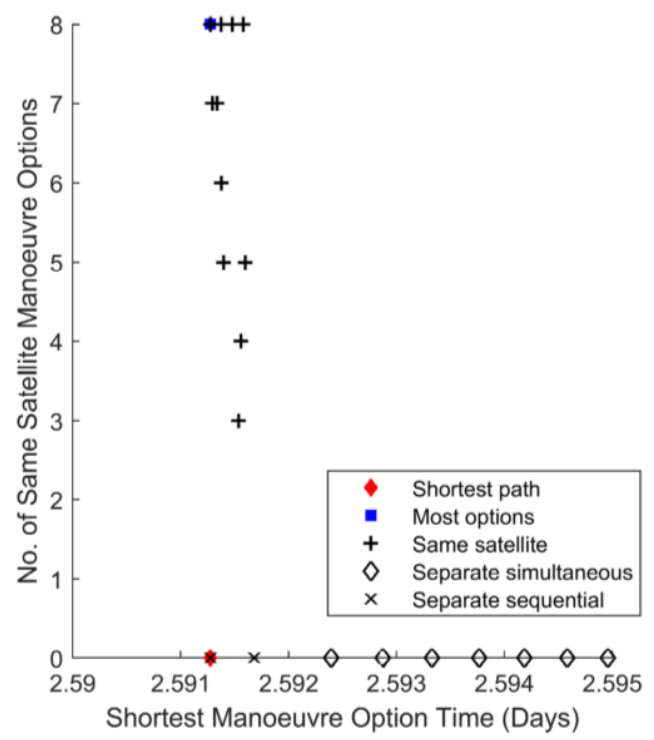

$\mathrm{b}$

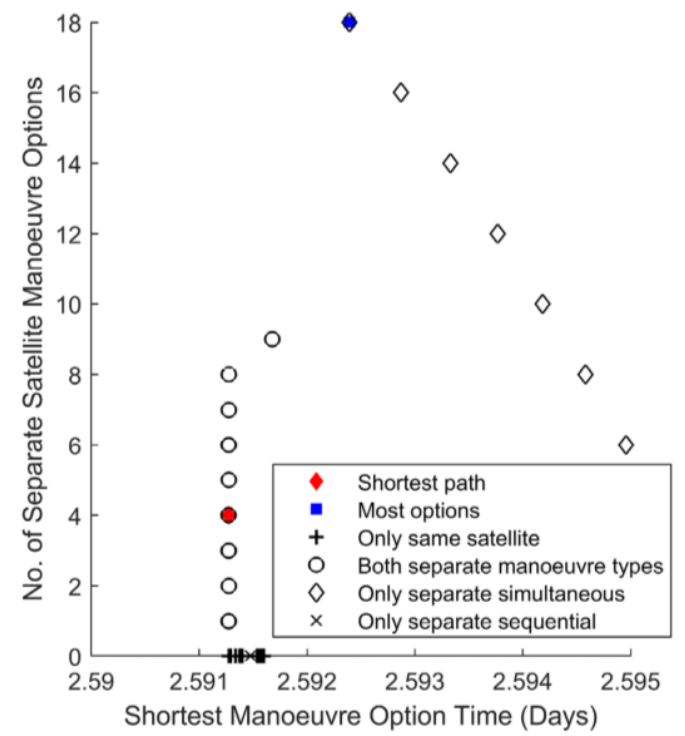

Figure 5: Shortest manoeuvre sequences for time, which complete both target flyovers within 10 minutes of the fastest flyover, alongside the number of manoeuvre options available in Stage $\mathbf{2}$ for (a) same satellite manoeuvres with markers indicating the type of manoeuvre producing the shortest time and (b) separate satellite manoeuvres with the markers indicating the type of manoeuvre options available after the stage 1 node.

\subsection{Minimum $\Delta \mathrm{V}$ Solution}

The shortest path solution, for $\Delta \mathrm{V}$, is one in which none of the satellites manoeuvre as the satellites naturally cover both targets in just over 3 days. A more interesting scenario emerges when constraining the graph so that the mission must be completed in under 3 days. This restriction produces a shortest path of $2.045 \mathrm{~m} / \mathrm{s} \Delta \mathrm{V}$, which includes $2 \mathrm{~m} / \mathrm{s}$ for active manoeuvring and an additional $0.045 \mathrm{~m} / \mathrm{s}$ for drag compensation. However, as in the minimum time case, the graph can provide greater insights than the shortest path alone when also restricting the total $\Delta \mathrm{V}$ to $5 \mathrm{~m} / \mathrm{s}$ or less, not including drag compensation.

Figure 6 displays a similar result to the minimum time scenario in Figure 5, where same satellite manoeuvre options are available that perform almost as well as the shortest path; though it should be noted that fewer options are available than seen in the minimum time scenario. As in the minimum time case, the shortest path has both separate simultaneous and separate sequential manoeuvres available, see Fig. 6, and hence could be an attractive option if ensuring target capture is a higher priority than reducing constellation disruption. 
a

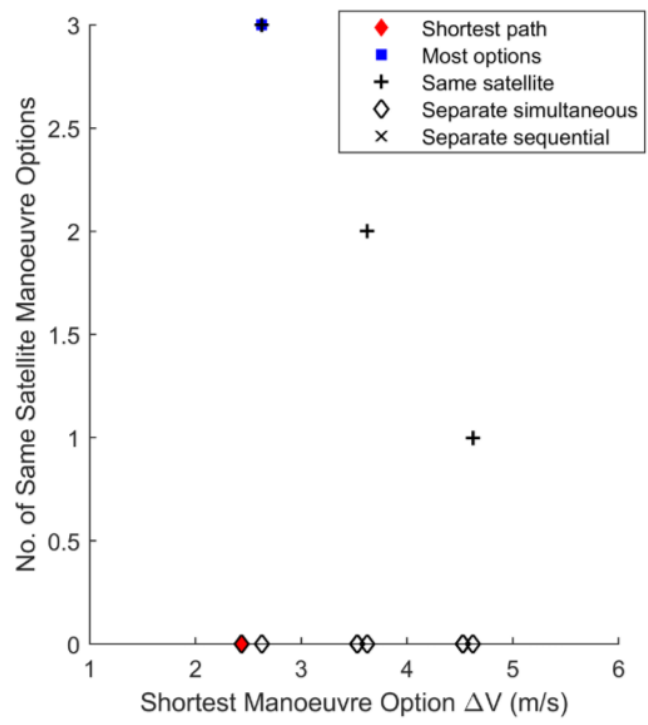

b

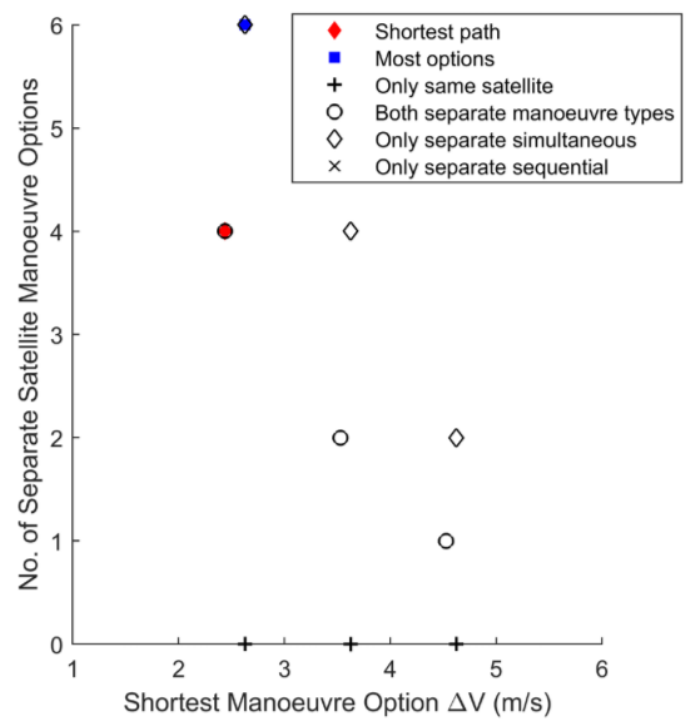

Figure 6: Shortest manoeuvre sequences for $\Delta V$, which complete both target flyovers with no more than $5 \mathrm{~m} / \mathrm{s} \Delta \mathrm{V}$, alongside the number of manoeuvre options available in Stage 2 for (a) same satellite manoeuvres with markers indicating the type of manoeuvre producing the shortest time and (b) separate satellite manoeuvres with the markers indicating the type of manoeuvre options available after the stage 1 node.

\section{RESULTS FOR REDUCED GRAPH}

This section focuses on the same scenario detailed in Section 3, but the graph is reduced. This demonstrates how the proposed method can be used for efficient mission design by enabling the effect of changes in mission parameters to be rapidly assessed by retraversing the graph. This process does not require individual manoeuvre options to be recalculated but instead generates a new graph by removing edges from the original graph. In this case only two satellites are considered, both in the same orbit plane, the swath width is reduced to 50 $\mathrm{km}$, as compared to the previously defined $100 \mathrm{~km}$, and only manoeuvres requiring $5 \mathrm{~m} / \mathrm{s} \Delta \mathrm{V}$ or less, excluding any drag compensation, are included.

\subsection{Minimum Time Solution}

The constraints on the $\Delta \mathrm{V}$ and swath width produce a smaller, more constrained, graph. Despite these changes, the shortest path remains similar, taking 2 days 14 hours and 13.3 minutes (2.5926 days). The main change is that there are no longer any same satellite manoeuvres that can achieve a fast time with the shortest same satellite sequence taking around 9 hours and 40 minutes longer. There are also no viable separate sequential manoeuvres, with separate simultaneous manoeuvres the only viable option for an operator looking for the fastest response.

\subsection{Minimum $\Delta \mathrm{V}$ Solution}

As in the full graph case, the targets can be reached without active manoeuvring. In this case, the same satellite would flyover both targets in 7 days 1 hour and 35 minutes. When considering the 3 day requirement for flyover of both targets, the shortest path is $4.048 \mathrm{~m} / \mathrm{s}$ $\Delta \mathrm{V}$ compared with the $2.045 \mathrm{~m} / \mathrm{s} \Delta \mathrm{V}$ possible in the full graph, due to the reduced swath width and fewer satellites. Figure 7 displays that the shortest path is the clear best choice as it 
is in possession of a comparatively large number of options after the Stage 1 manoeuvre, with eight in total (four $\Delta \mathrm{V}$ manoeuvres requiring $4 \mathrm{~m} / \mathrm{s}$ and four requiring $5 \mathrm{~m} / \mathrm{s}$ ). However, as mentioned in Section 5.1, this same satellite sequence takes longer than the separate satellite options with a time of 2 days 23 hours and 54 minutes.

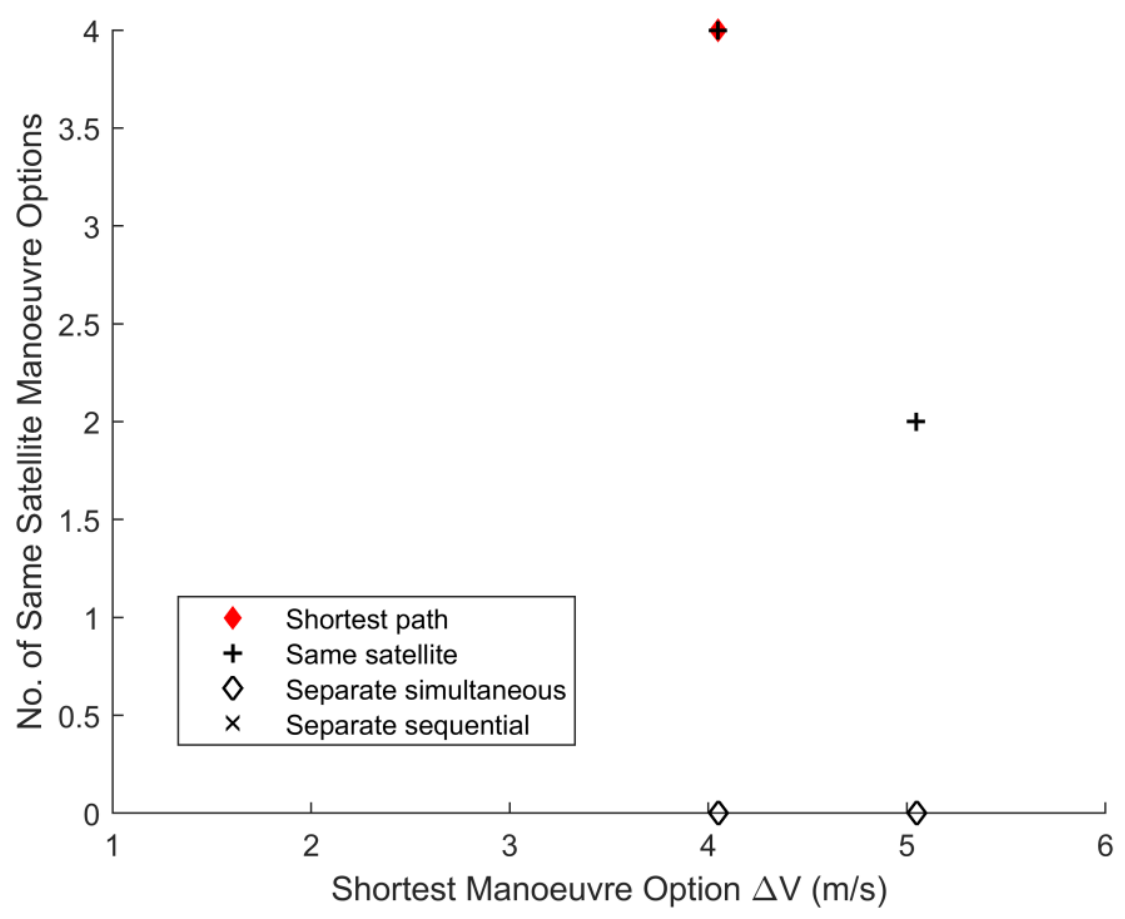

Figure 7: Shortest manoeuvre sequences for $\Delta \mathrm{V}$, which complete both target flyovers with no more than $5 \mathrm{~m} / \mathrm{s} \Delta \mathrm{V}$ for the reduced graph, alongside the number of same satellite manoeuvre options available in Stage 2 with markers indicating the type of manoeuvre producing the shortest time.

\section{CONCLUSIONS}

Analysing responsive spacecraft manoeuvres using graph techniques allows designers and operators to consider the full responsive mission, rather than just considering an individual manoeuvre that may be initially optimal but result in a poorer performance over the whole operational scenario. The graph captures not only the best sequence of manoeuvres but also presents the operator with a choice between optimality and resilience/redundancy. An initial manoeuvre can be identified as resilient if the same satellite can accomplish the mission for a wider range of $\Delta \mathrm{V}$ inputs. An operator can also ensure redundancy by identifying manoeuvre sequences where other satellites can fulfil the mission if tasked simultaneously or subsequently. The use of the graph highlighted that, for the cases considered, a same satellite manoeuvre could reduce constellation disruption while still covering both targets within a second of the fastest time possible by manoeuvring two satellites. The presented technique, therefore, provides a method of analysing the performance of responsive constellations in operational scenarios. 


\section{REFERENCES}

Bate, R. R., Mueller, D. D. \& White, J. E., 1971. Fundamentals of Astrodynamics. New York: Dover Publications.

Boshuizen, C., Mason, J., Klupar, P. \& Spanhake, S., 2014. Results from the Planet Labs flock constellation. Presented at the AIAA/USU Small Satellite Conference, Logan, Utah.

Buchen, E., 2015. Small satellite market observations. Presented at the AIAA/USU Small Satellite Conference, Logan, Utah.

Carver, S., Comber, L., Fritz, S., McMorran, R., Taylor, S., \& Washtell, J., 2008. Wildness study in the Cairngorms National Park, Technical Report: University of Leeds.

Chu, X., Chen, Y. \& Tan, Y., 2017. An anytime branch and bound algorithm for agile earth observation satellite onboard scheduling. Advances in Space Research, 60(9), pp.2077-2090. doi: 10.1016/j.asr.2017.07.026

Dijkstra, E. W., 1959. A note on two problems in connexion with graphs. Numerische Mathematic (Numerical Mathematics), 1, pp. 269-271.

Gnawali, O., Polyakovt, M., Bose, P. \& Govindan, R., 2005. Data centric, position-based routing in space networks. Proceedings of the 2005 IEEE Aerospace Conference, pp. 13221334. doi: 10.1109/AERO.2005.1559422

Gopinath, G., 2015. Free data and Open Source Concept for Near Real Time Monitoring of Vegetation Health of Northern Kerala, India. Aquatic Procedia, 4, pp. 1461-1468. doi: 10.1016/j.aqpro.2015.02.189

Gray, A. \& Levy, P., 2009. A review of carbon flux research in UK peatlands in relation to fire and the Cairngorms National Park, Technical Report: Natural Environment Research Council Centre for Ecology \& Hydrology.

Kane, V. R., North, M. P., Lutz, J. A., Churchill, D. J., Roberts, S. L., Smith, D. F., McGaughey, R. J., Kane, J. T. \& Brooks, M. L., 2014. Assessing fire effects on forest spatial structure using a fusion of Landsat and airborne LiDAR data in Yosemite National Park. Remote Sensing of Environment, 151, pp. 89-101. doi: 10.1016/j.rse.2013.07.041

Krejci, D., Mier-Hicks, F., Thomas, R., Haag, T. \& Lozano, P., 2017. Emission characteristics of passively fed electrospray microthrusters with propellant reservoirs. Journal of Spacecraft and Rockets, 54(2), pp. 447-458. doi: 10.2514/1.A33531

Lowe, C. \& Macdonald, M., 2016. Resource-Considerate Data Routing Through Satellite Networks. Journal of Aerospace Information Systems, 14(8), pp. 472-482. doi: 10.2514/1.I010423

Lutz J. A., van Wagtendonk J. W., Thode A. E., Miller J. D. \& Franklin J. F. 2009. Climate, lightning ignitions, and fire severity in Yosemite National Park, California, USA. International Journal of Wildland Fire, 18(7), pp. 765-774. doi:10.1071/WF08117

McGrath, C. N., 2018. Analytical Methods for Satellite Constellation Reconfiguration and Reconnaissance using Low-Thrust Manoeuvres, $\mathrm{PhD}$ : Mechanical and Aerospace Engineering, University of Strathclyde.

McGrath, C. N. \& Macdonald, M., 2019. General Perturbation Method for Satellite Constellation Reconfiguration using Low-Thrust Maneuvers. Journal of Guidance, Control and Dynamics, 42(8), pp. 1676-1692. doi: 10.2514/1.G003739

Mier-Hicks, F. \& Lozano, P., 2017. Electrospray Thrusters as Precise Attitude Control Actuators for Small Satellites. Journal of Guidance, Control, and Dynamics, 40(3), pp. 642649. doi:10.2514/1.G000736 
Morin, J., 2019. Four steps to global management of space traffic. Nature, 567, pp. 25-27. doi: 10.1038/d41586-019-00732-7

Morrison, D.R., Jacobson, S.H., Sauppe, J.J. \& Sewell, E.C., 2016. Branch-and-bound algorithms: A survey of recent advances in searching, branching, and pruning. Discrete Optimization, 19, pp.79-102. doi: 10.1016/j.disopt.2016.01.005

National Park Service, 2017. National Park Service visitor use statistics for Yosemite National Park. [Online] Available at: https://irma.nps.gov/Stats/Reports/Park/YOSE [Accessed 9 February 2018].

Platzer, P., Wake, C. \& Gould, L., 2015. Smaller Satellites, Smarter Forecasts: GPS-RO Goes Mainstream. Presented at the AIAA/USU Small Satellite Conference, Logan, Utah.

Santilli, G., Vendittozzi, C., Cappelletti, C., Battistini, S., \& Gessini, P., 2018. CubeSat constellations for disaster management in remote areas. Acta Astronautica, 145, pp. 11-17. doi: 10.1016/j.actaastro.2017.12.050

Silver, D., Huang, A., Maddison, C. et al., 2016. Mastering the game of Go with deep neural networks and tree search. Nature 529, pp. 484-489. doi: 10.1038/nature16961

Skinner, M. A., Jah, M. K., McKnight, D., Howards, D., Murakami, D. \& Schrogl, K., 2019. Results of the international association for the advancement of space safety space traffic management working group. Journal of Space Safety Engineering, 6(2), pp. 88-91. doi: 10.1016/j.jsse.2019.05.002

Voigt, S., Giulio Tonolo, F., Lyons, J., Kucera, J., Jones, B., Schneiderhan, T., Platzeck, G., Kaku, K., Hazarika, M., Czaran, L., Li, S., Pedersen, W., James, G., Proy, C., Macharia, D., Bequignon, J. \& Guha-Sapir, D., 2016. Global trends in satellite-based emergency mapping. Science, 353(6296), pp. 247-252. doi:10.1126/science.aad8728

Walker, J. G., 1977. Continuous Whole-Earth Coverage by Circular-Orbit Satellite Patterns, Farnborough, UK: Royal Aircraft Establishment. 